\title{
OPEN Graphene optical modulators using bound states in the continuum
}

\begin{abstract}
Myunghwan Kim${ }^{1}$, Sangin $\mathrm{Kim}^{2 \bowtie}$ \& Soeun $\mathrm{Kim}^{1 \bowtie}$
Graphene-based optical modulators have been widely investigated due to the high mobility and tunable permittivity of graphene. However, achieving a high modulation depth with a low insertion loss is challenging owing to low graphene-light interaction. To date, only waveguide-type modulators have been extensively studied to improve light-graphene interaction, and few free-space type modulators have been demonstrated in the optical communication wavelength range. In this study, we propose two graphene-based optical free-space type modulators in a simple silicon photonic crystal structure that supports bound states in the continuum. The designed modulator with an ultra-high quality factor from the bound states in the continuum achieves a high modulation depth ( $M D=0.9972)$ and low insertion loss $(I L=0.0034)$ with a small Fermi level change at the optical communication wavelength. In addition, the proposed modulators support outstanding modulation performance in the normal chemical vapor deposition (CVD) graphene (mobility $=0.5 \mathrm{~m}^{2} / \mathrm{Vs}$ ). We believe the scheme may pave the way for graphene-based optical active devices.
\end{abstract}

Graphene, where a single layer of atoms is arranged in a two-dimensional honeycomb lattice, has gained considerable attentions owing to its various exceptional properties, such as its high thermal conductivity, ultra-high saturable absorption, and wide optical bandwidth ${ }^{1-3}$. Numerous graphene-based optical devices have been studied for many years; these include photo detectors ${ }^{4-7}$, optical absorbers ${ }^{8-11}$, and nonlinear devices ${ }^{12-15}$. In particular, graphene-based optical modulators, one of the key components of photonics systems, have been extensively investigated. The outstanding carrier mobility and gate-tunable carrier concentration of graphene enable it to be used as an active medium in optical modulators; the carrier concentrations of graphene can be tuned by applying different gate-voltage (Fermi level variation), which enables gate-tunable absorption of graphene ${ }^{16}$.

To date, many waveguide and free-space optical modulators using graphene loss variation have been proposed over a wide wavelength range. Optical transitions, including interband and intraband transitions, are the main processes that determine the loss of graphene. In the terahertz and mid-infrared wavelength regions, where intraband transition is dominant, absorption in graphene is largely tuned by adjusting the drive voltage. Therefore, many highly efficient graphene-based optical modulators have been demonstrated in these wavelength regions, such as optical modulators using metal reflectors ${ }^{17}$, graphene metamaterials ${ }^{18-21}$, graphene antennas ${ }^{22}$. In contrast, graphene loss is largely determined by the interband transition in the optical communication wavelength region, which is nearly independent of the Fermi level of graphene. Therefore, waveguide-type modulators have been extensively studied to improve light-graphene interaction in this wavelength range. A graphene-coated Si waveguide-type optical modulator operating in the optical communication wavelength was the first to be experimentally demonstrated ${ }^{23}$. This device had a small device area $\left(\sim 25 \mu \mathrm{m}^{2}\right)$ with a high modulation depth $(\mathrm{MD}=0.1 \mathrm{~dB} / \mu \mathrm{m})$, compared to conventional Si-based optical modulators. Subsequently, double graphene layer-coated waveguide-type optical modulators ${ }^{24,25}$, suspended graphene modulators ${ }^{26}$, and hybrid graphene modulators ${ }^{27}$ have been demonstrated with improved the modulator performance. However, waveguide-type modulators have a limited range of applications because they exhibit a trade-off between modulation depth and insertion loss (IL). In particular, they are not suitable for free-space applications that require a low insertion loss. Lee et al. proposed a free-space graphene modulator composed of a quarter-wavelength-thick insulator layer and a metal reflector to increase graphene absorption by placing the graphene layer where the amplitude of the electric field was maximized ${ }^{28}$. However, this modulator showed a very low modulation depth of $4 \%$ owing to the low absorption of graphene (2.3\% absorption for normal incidence).

To increase absorption in graphene, optical modulators using the epsilon-near-zero effect have been introduced $^{29,30}$. These devices achieved a high modulation depth with an extremely enhanced electric field in the graphene layer. However, the epsilon-near-zero effect in graphene is highly debated and has not been experimentally demonstrated. Another approach to increase light-graphene interaction is to place a graphene layer

\footnotetext{
${ }^{1}$ Integrated Optics Laboratory, Advanced Photonics Research Institute, Gwangju Institute of Science and Technology, Gwangju 61005, South Korea. ${ }^{2}$ Department of Electrical and Computer Engineering, Ajou University, Suwon 16499, South Korea. ${ }^{\bowtie}$ email: sangin@ajou.ac.kr; sekim@gist.ac.kr
} 
(a)

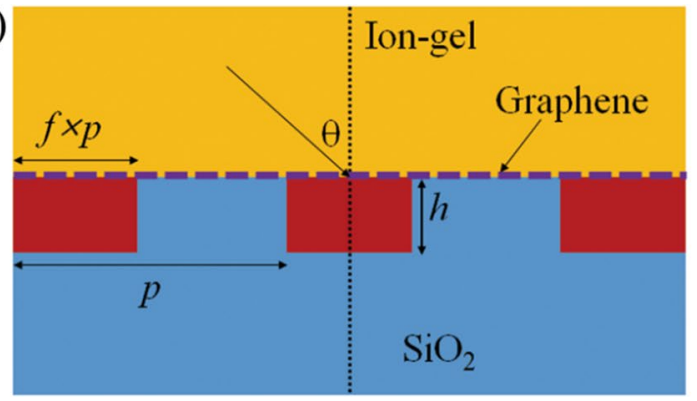

(b)

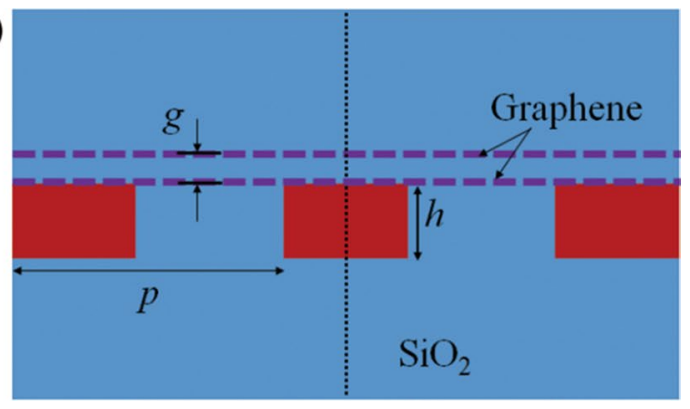

Figure 1. Schematic diagrams of the graphene-based optical modulators. (a) Ion gel and single-layer graphene modulator and (b) double-layer graphene modulator.

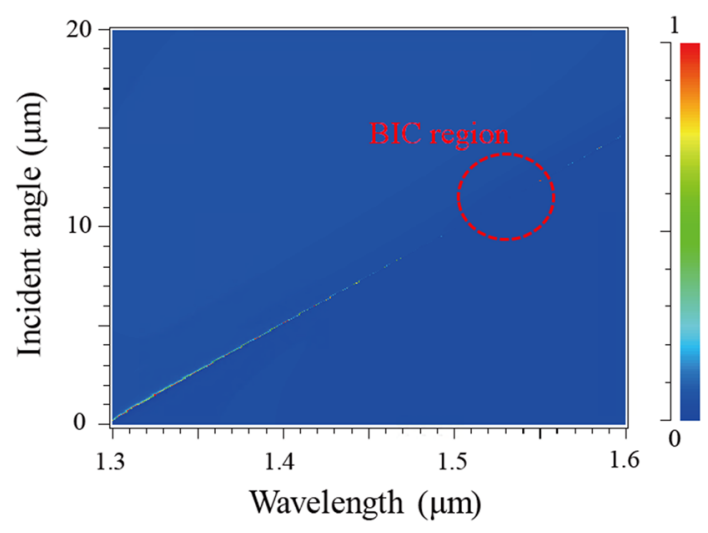

Figure 2. Reflection spectrum as a function of the incident angle: $p=870 \mathrm{~nm}, h=150 \mathrm{~nm}$, and $f=0.3$.

near high Q-factor resonators, such as photonic crystal resonators ${ }^{31}$ and whispering gallery mode ${ }^{32,33}$. However, these resonators show relatively low $\mathrm{Q}$ values $\left(\mathrm{Q}=10^{3} \sim 10^{4}\right)$, which is not enough to achieve highly efficient modulators; Scattering loss by imperfect fabrication and graphene loss deteriorate the $\mathrm{Q}$ value.

In this study, we propose two graphene-based optical modulators using bound states in the continuum (BICs) in a simple one-dimensional photonic crystal structure. Theoretically, BICs support infinite Q resonance as a result of destructive interference between radiative waves, and they can be easily obtained from photonic crystal structures $^{34-40}$. In addition, high $\mathrm{Q}\left(>10^{5}\right)$ is maintained in the symmetry broken structures due to imperfect fabrication: tiled, imperfectly etched, or bent structure ${ }^{34}$. In the proposed scheme, the high $\mathrm{Q}$ transmission resonance from the BIC facilitates a sharp transmission variation from the tuning of the Fermi level of graphene, which enables the proposed modulators to simultaneously achieve a very high modulation depth and low insertion loss. We also investigate the effect of graphene mobility on modulation efficiency. The proposed modulators maintain high performance if the graphene mobility is higher than $\mu=0.5 \mathrm{~m}^{2} / \mathrm{Vs}$, and this value can be obtained from normal graphene.

\section{Results}

Structure of the modulators. Figure 1a,b show schematics of the proposed optical modulators composed of ion gel and single-layer graphene (ISLG), and double-layer graphene (DLG), respectively. In the figures, $p$ is the period, $f$ is the fill factor, $h$ is the height of $\mathrm{Si}$, and $\theta$ is the incident angle. The refractive indices of $\mathrm{Si}$, ion gel, and $\mathrm{SiO}_{2}$ are 3.45, 1.45 and 1.45, respectively. The permittivity of graphene is calculated from the Kubo formula $\mathrm{a}^{41}$, and the height of the graphene layer is assumed to be $0.34 \mathrm{~nm}$. Ion gel is used for the electrical doping of graphene in the ISLG modulator structure. Note that there are many kinds of ion-gel materials. The refractive index of most of the ion gels is in the range of 1.4 1.45, and IL-P14 and IL-AP3 have the refractive index of $1.45^{42-44}$. In recent years, ion-gel materials have been actively utilized as an efficient gating medium. They have great advantages such as transparent, good mechanical flexibility, thermal stability, easy fabrication, and compatibility with various substrates. The electric double layer with extremely high capacitance at the grapheneion gel interface enables the chemical potential (Fermi level) of graphene to be adjusted with a low electric gate voltage $^{45-47}$. In the DLG structure, graphene doping is implemented by applying a gate voltage between two graphene layers.

First, we designed a photonic crystal structure to induce a BIC phenomenon. Figure 2 shows the reflection spectrum as a function of the incident angle in the proposed photonic crystal structure without graphene layers. We used the following parameters: $p=870 \mathrm{~nm}, h=150 \mathrm{~nm}$, and $f=0.3$. The parameters were selected to induce 

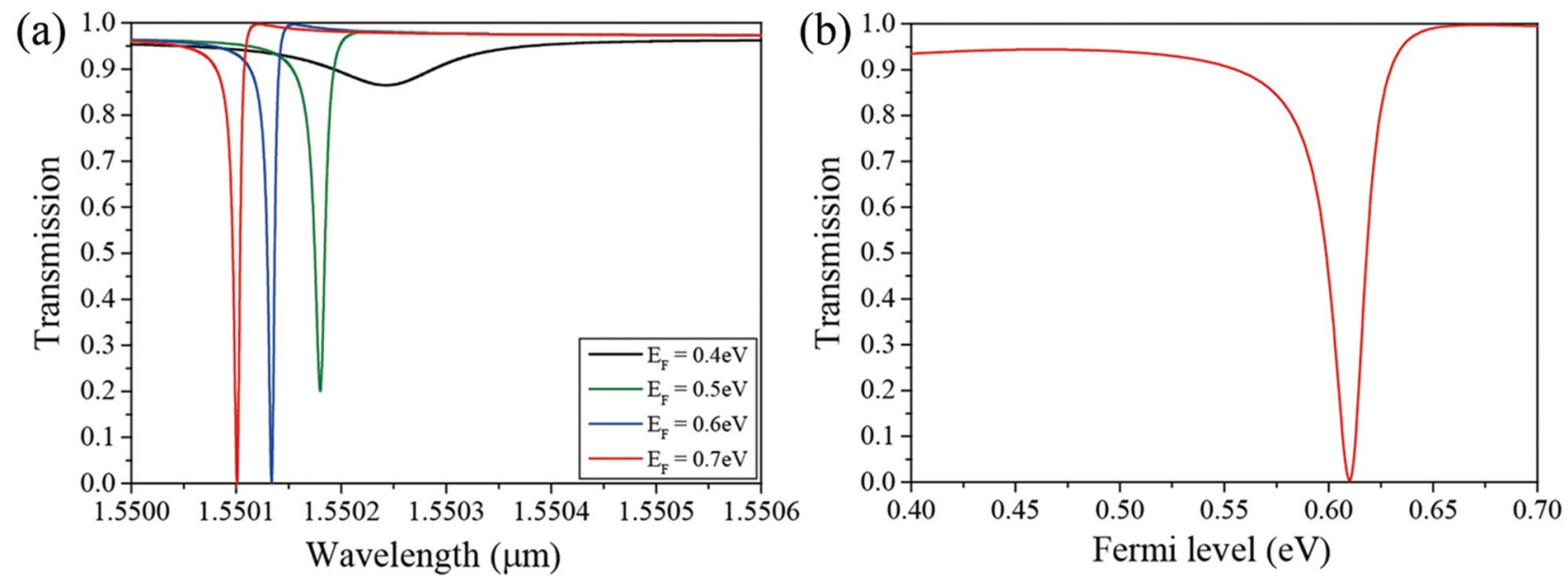

Figure 3. (a) Transmission spectra for Fermi level variation from $\mathrm{E}_{\mathrm{F}}=0.4 \mathrm{eV}$ to $0.7 \mathrm{eV}$. (b) Transmission variation as a function of the Fermi level in the SLG modulator. The mobility of graphene is $\mu=1 \mathrm{~m}^{2} / \mathrm{Vs}$.

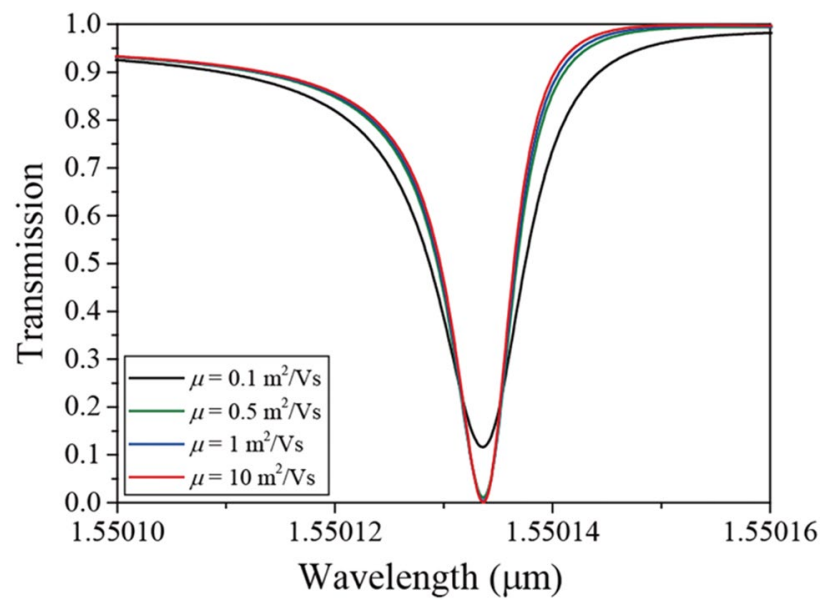

Figure 4. Transmission spectra for graphene mobility variation from $\mu=0.1$ to $\mu=10 \mathrm{~m}^{2} / \mathrm{Vs}$ for $\mathrm{E}_{\mathrm{F}}=0.6 \mathrm{eV}$.

the BIC phenomenon near the operating wavelength. The BIC phenomenon is observed near $\lambda=1.54 \mu \mathrm{m}$ and $\theta=12^{\circ}$, and the reflection peak (transmission dip) disappears in this region due to the infinite Q because of BIC.

We investigated the variation in the transmission spectra for Fermi level variation in the proposed ISLG structure. The mobility of graphene is assumed to be $\mu=1 \mathrm{~m}^{2} / \mathrm{Vs}$. In this case, the BIC disappears owing to the graphene loss; $\mathrm{Q}$ is inversely proportional to the loss, and the loss of graphene breaks the infinite $\mathrm{Q}$ condition of the BIC. However, an ultra-high Q is maintained because the loss of doped graphene is insignificant, which facilitates the design of high-performance modulators. Figure 3 a shows the transmission spectra of the ISLG for the Fermi level variation from $\mathrm{E}_{\mathrm{F}}=0.4 \mathrm{eV}$ to $0.7 \mathrm{eV}$ at the incident angle of $\theta=11.937^{\circ}$. By choosing the incident angle slightly apart from the BIC, the transmission resonance with ultra-high Q at the operating wavelength can be achieved. The other parameters are assumed to be the same as in the previous calculations. As increase in the Fermi level leads to a decrease in the graphene loss, the $\mathrm{Q}$ of the transmission dip increases with an increase in the Fermi level. The $\mathrm{Q}$ of the resonance for $\mathrm{E}_{\mathrm{F}}=0.7 \mathrm{eV}$ is $\mathrm{Q} \sim 2 \times 10^{5}$, and it can be simply increased by adjusting the incident angle. In addition, it has been shown that increasing the Fermi level of graphene leads to a transmission dip blue shift because the permittivity of graphene decreases as the Fermi level increases. Generally, graphene doping (Fermi level variation) has little effect on tuning the resonant wavelength in resonators because the variation of the graphene permittivity does not significantly change the effective index of the structure due to the very thin graphene. However, in this ultra-high Q resonance system, a small resonant wavelength tuning results in a very high transmission variation. The transmission variation for the Fermi level variation at $\lambda=1.55013 \mu \mathrm{m}$ is shown in Fig. $3 b$. The transmission becomes approximately zero and one at $E_{F}=0.615$ (off-state) and $E_{F}=0.672 \mathrm{eV}$ (on-state), respectively. Therefore, a very high modulation efficiency was achieved: $\mathrm{MD}=0.9972$ and $\mathrm{IL}=0.0034$, where the modulation depth was obtained as $\mathrm{MD}=\left(\mathrm{T}_{\mathrm{on}}-\mathrm{T}_{\text {off }}\right) / \mathrm{T}_{\text {on }}$, and insertion loss, which is ratio of the transmitted power for on-state and input power, was calculated as $I L=1-T_{\text {on }}$.

Figure 4 shows the transmission spectra for the mobility variation from $\mu=0.1 \mathrm{~m}^{2} / \mathrm{Vs}$ to $\mu=10 \mathrm{~m}^{2} / \mathrm{Vs}$ for $\mathrm{E}_{\mathrm{F}}=0.6 \mathrm{eV}$. Because the mobility is inversely proportional to the loss, a higher $\mathrm{Q}$ is observed for a higher graphene 

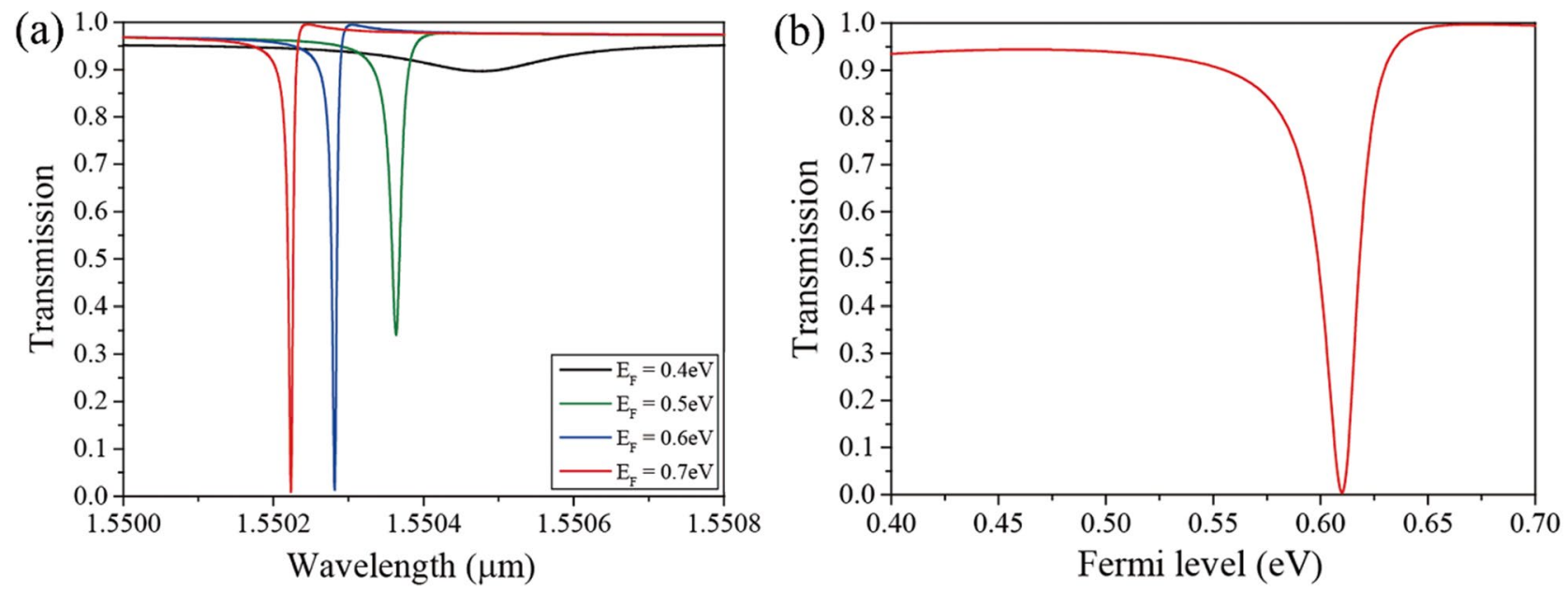

Figure 5. (a) Transmission spectra for Fermi level variation from $\mathrm{E}_{\mathrm{F}}=0.4 \mathrm{eV}$ to $0.7 \mathrm{eV}$. (b) Transmission variation as a function of the Fermi level in the DLG modulator. The mobility of graphene is $\mu=1 \mathrm{~m}^{2} / \mathrm{Vs}$.

mobility. However, the high Q is maintained even though the mobility is $\mu=0.1 \mathrm{~m}^{2} / \mathrm{Vs}$, and the transmission spectra for $\mu>0.5 \mathrm{~m}^{2} / \mathrm{Vs}$ are almost the same. Therefore, a high modulation efficiency can be sustained for $\mu>0.5$ $\mathrm{m}^{2} /$ Vs. Note that the real part of graphene permittivity is almost constant for the mobility variation. Therefore, the resonant wavelength hardly shifts for the mobility variation. In addition, as the mobility increases, the imaginary part of the graphene permittivity sharply decreases in the range of $\mu=0 \sim 0.5 \mathrm{~m}^{2} / \mathrm{Vs}$. Therefore, the transmission spectra largely change when the mobility is varied from $\mu=0.1$ to $0.5 \mathrm{~m}^{2} / \mathrm{Vs}$. It should be noted that a mobility of $\mu=0.5 \mathrm{~m}^{2} /$ Vs can be obtained from the chemical vapor deposition (CVD) method that is considered the most promising method for producing graphene $\mathrm{e}^{48,49}$.

Although the ion gel-based modulator shows outstanding modulation performance, the low stability and slow modulation speed of the ion gel are obstacles to realizing high-speed optical modulators. To solve these problems, we designed an optical modulator composed of two graphene layers (Fig. 1b). By applying a gate voltage between the two graphene layers, stable high-speed graphene doping is possible. Figure 5 a shows the transmission spectra for the Fermi level variation. The mobility of graphene is assumed to be $\mu=1 \mathrm{~m}^{2} / \mathrm{Vs}$. The $\mathrm{Q}$ of the resonance is slightly reduced compared to that of ISLG owing to the multiplied graphene loss. However, this modulator also supports a very high $\mathrm{Q}$ transmission. The transmission variation for the Fermi level at an operating wavelength of $\lambda=1.55028 \mu \mathrm{m}$ is illustrated in Fig. 5b. The calculated modulation depth and insertion loss are approximately $\mathrm{MD}=0.9889$ and $\mathrm{IL}=0.011$, respectively. The modulation depth was slightly reduced, and the insertion loss slightly increased. However, very high modulation performances are maintained.

\section{Conclusions}

In this study, we proposed two graphene-based free-space high-performance optical modulators with a simple photonic crystal structure. The very high Q transmission resonance from BIC facilitated a remarkable transmission change with a small variation in the Fermi level. The $\mathrm{Q}$ of the proposed structure is approximate $\sim 2 \times 10^{5}$, and this value is about ten times higher than previous reported graphene-based resonators. In addition, the $\mathrm{Q}$ can increase easily by tuning the incident angle. The proposed modulators could simultaneously support a high modulation depth and low insertion loss: $\mathrm{MD}_{\mathrm{ISLG}}=0.9972, \mathrm{IL}_{\mathrm{ISLG}}=0.0034, \mathrm{MD}_{\mathrm{ISLG}}=0.9889$, and $\mathrm{IL}_{\mathrm{ISLG}}=0.011$. The effect of graphene mobility on the performance of the modulators was also investigated. Although low graphene mobility $\left(\mu=0.1 \mathrm{~m}^{2} / \mathrm{Vs}\right)$ deteriorated the efficiency of the modulator, an outstanding efficiency could be maintained for standard quality graphene $\left(\mu>0.5 \mathrm{~m}^{2} / \mathrm{Vs}\right)$. These promising features are likely to generate new avenues for graphene-based optical devices for free-space applications.

\section{Methods}

The permittivity of graphene was calculated using the Kubo formula, assuming a graphene thickness of $0.34 \mathrm{~nm}$ and Fermi velocity of $10^{6} \mathrm{~m} / \mathrm{s}$. The reflection spectrum of the photonic crystal structure without graphene as a function of the incident angle was calculated using rigorous coupled-wave analysis (RCWA), and the transmission spectra of the modulators were calculated using the COMSOL Multiphysics software. Periodic boundary condition was used for the calculations, and a sufficiently small mesh size was used for accurate calculations.

Received: 8 November 2021; Accepted: 5 January 2022

Published online: 27 January 2022

\section{References}

1. Geim, A. K. \& Novoselov, K. S. The rise of graphene PROGRESS. Nat. Mater. 6, 183-191 (2007).

2. Novoselov, K. S. et al. Electric field effect in atomically thin carbon films. Science 306, 666-669 (2016).

3. Bonaccorso, F., Sun, Z., Hasan, T. \& Ferrari, A. C. Graphene photonics and optoelectronics. Nat. Photonics 4, 611-622 (2010). 
4. Mueller, T., Xia, F. \& Avouris, P. Graphene photodetectors for high-speed optical communications. Nat. Photonics 4, 297-301 (2010).

5. Guo, X. et al. High-performance graphene photodetector using interfacial gating. Optica 3, 1066-1070 (2016).

6. Gosciniak, J., Rasras, M. \& Khurgin, J. B. Ultrafast plasmonic graphene photodetector based on the channel photothermoelectric effect. ACS Photonics 7, 488-498 (2020).

7. Liu, C. H., Chang, Y. C., Norris, T. B. \& Zhong, Z. Graphene photodetectors with ultra-broadband and high responsivity at room temperature. Nat. Nanotechnol. 9, 273-278 (2014).

8. Alaee, R., Farhat, M., Rockstuhl, C. \& Lederer, F. A perfect absorber made of a graphene micro-ribbon metamaterial. Opt. Express 20, 28017-28024 (2012).

9. Andryieuski, A. \& Lavrinenko, A. V. Graphene metamaterials based tunable terahertz absorber: Effective surface conductivity approach. Opt. Express 21, 9144-9155 (2013).

10. Lee, S., Tran, T. Q., Heo, H., Kim, M. \& Kim, S. A proposal of a perfect graphene absorber with enhanced design and fabrication tolerance. Sci. Rep. 7, 4760 (2017).

11. Lee, S. et al. Angle- and position-insensitive electrically tunable absorption in graphene by epsilon-near-zero effect. Opt. Express 23, 33350-33358 (2015).

12. Bao, Q. et al. Atomic-layer craphene as a saturable absorber for ultrafast pulsed lasers. Adv. Funct. Mater. 19, 3077-3083 (2009),

13. Cox, J. D. \& García De Abajo, F. J. Nonlinear graphene nanoplasmonics. Acc. Chem. Res. 52, 2536-2547 (2019).

14. Jiang, T., Kravtsov, V., Tokman, M., Belyanin, A. \& Raschke, M. B. Ultrafast coherent nonlinear nanooptics and nanoimaging of graphene. Nat. Nanotechnol. 14, 838-843 (2019).

15. Kim, M., Kim, S. \& Kim, S. Resonator-free optical bistability based on epsilon-near-zero mode. Sci. Rep. 9, 6552 (2019).

16. Wang, F. et al. Gate-variable optical transitions in graphene. Science 320, 206-209 (2008).

17. Sensale-Rodriguez, B. et al. Extraordinary control of terahertz beam reflectance in graphene electro-absorption modulators. Nano Lett. 12, 4518-4522 (2012).

18. Zhu, W., Rukhlenko, I. D. \& Premaratne, M. Graphene metamaterial for optical reflection modulation. Appl. Phys. Lett. 102, 4-7 (2013).

19. Gao, W. et al. High-contrast terahertz wave modulation by gated graphene enhanced by extraordinary transmission through ring apertures. Nano Lett. 14, 1242-1248 (2014).

20. Ju, L. et al. Graphene plasmonics for tunable terahertz metamaterials. Nat. Nanotechnol. 6, 630-634 (2011).

21. Kim, M., Kim, S. \& Kim, S. Ultra-compact integrated terahertz modulator based on a graphene metasurface. Opt. Lett. 46, 605-608 (2021).

22. Li, Z. \& Yu, N. Modulation of mid-infrared light using graphene-metal plasmonic antennas. Appl. Phys. Lett. 102, 131108 (2013).

23. Liu, M. et al. A graphene-based broadband optical modulator. Nature 474, 64-67 (2011).

24. Liu, M., Yin, X. \& Zhang, X. Double-layer graphene optical modulator. Nano Lett. 12, 1482-1485 (2012).

25. Koestera, S. J. \& Lib, M. High-speed waveguide-coupled graphene- on-graphene optical modulators. Appl. Phys. Lett. 100, 171107 (2012).

26. Liu, J., Khan, Z. U. \& Sarjoghian, S. Suspended graphene double-layer modulator with an ultrahigh figure of merit and a subwavelength-thickness modulator with leaky mode. Appl. Opt. 58, 3729-3734 (2019).

27. Ansell, D. et al. Hybrid graphene plasmonic waveguide modulators. Nat. Commun. 6, 8846 (2015).

28. Lee, C.-C., Suzuki, S., Xie, W. \& Schibli, T. R. Broadband graphene electro-optic modulators with sub-wavelength thickness. Opt. Express 20, 5264-5269 (2012).

29. Lu, Z., Zhao, W. \& Shi, K. Ultracompact electroabsorption modulators based on tunable epsilon-near-zero-slot waveguides. IEEE Photonics J. 4, 735-740 (2012).

30. Kim, M., Jeong, C. Y., Heo, H. \& Kim, S. Optical reflection modulation using surface plasmon resonance in a graphene-embedded hybrid plasmonic waveguide at an optical communication wavelength. Opt. Lett. 40, 871-874 (2015).

31. Gan, X. et al. High-contrast electrooptic modulation of a photonic crystal nanocavity by electrical gating of graphene. Nano Lett. 13, 691-696 (2013).

32. Phare, C. T., Daniel Lee, Y. H., Cardenas, J. \& Lipson, M. Graphene electro-optic modulator with $30 \mathrm{GHz}$ bandwidth. Nat. Photonics 9, 511-514 (2015).

33. Wang, L., Zhou, X., Yang, S., Huang, G. \& Mei, Y. 2D-material-integrated whispering-gallery-mode microcavity. Photonics Res. 7, 905-916 (2019).

34. Hsu, C. W. et al. Observation of trapped light within the radiation continuum. Nature 499, 188-191 (2013).

35. Hsu, C. W., Zhen, B., Stone, A. D. \& Joannopoulos, J. D. Bound states in the continuum. Nat. Rev. Mater. 1, 1-13 (2016).

36. Hsu, C. W. et al. Bloch surface eigenstates within the radiation continuum. Light Sci. Appl. 2, e84 (2013).

37. Sadrieva, Z. F. \& Bogdanov, A. A. Bound state in the continuum in the one-dimensional photonic crystal slab. J. Phys. Conf. Ser. 741, 012122 (2016).

38. Bulgakov, E. N. \& Maksimov, D. N. Propagating bound states in the continuum in dielectric gratings. J. Opt. Soc. Am. B 35 , 1218-1222 (2018).

39. Xiao, Y. X., Ma, G., Zhang, Z. Q. \& Chan, C. T. Topological subspace-induced bound state in the continuum. Phys. Rev. Lett. 118, 1-5 (2017).

40. Plotnik, Y. et al. Experimental observation of optical bound states in the continuum. Phys. Rev. Lett. 107, 28-31 (2011).

41. Emani, N. K. et al. Electrically tunable plasmonic resonances with graphene. Nano Lett. 12, 5202-5206 (2012).

42. Yamada, T. \& Otomo, A. Optimization of microscope unit for studying fluorescence emitters under high-vacuum and ambient gas conditions: Optical properties for various ionic liquids as a refractive index matching medium. Phys. Procedia 14, 7-11 (2011).

43. $\mathrm{Wu}, \mathrm{X}$. et al. Determination of the refractive indices of ionic liquids by ellipsometry, and their application as immersion liquids. Appl. Opt. 57, 9215 (2018).

44. Arosa, Y. et al. Refractive index measurement of imidazolium based ionic liquids in the Vis-NIR. Opt. Mater. 73, 647-657 (2017).

45. Kim, U. J. et al. Modulation of the Dirac point voltage of graphene by ion-gel dielectrics and its application to soft electronic devices. ACS Nano 9, 602-611 (2015).

46. Kim, J. T., Choi, H., Choi, Y. \& Cho, J. H. Ion-gel-gated graphene optical modulator with hysteretic behavior. ACS Appl. Mater. Interfaces 10, 1836-1845 (2018).

47. Hu, H. et al. Broadly tunable graphene plasmons using an ion-gel top gate with low control voltage. Nanoscale 7, 19493-19500 (2015).

48. Chen, B. et al. How good can CVD-grown monolayer graphene be?. Nanoscale 6, 15255-15261 (2014).

49. Banszerus, L. et al. Ultrahigh-mobility graphene devices from chemical vapor deposition on reusable copper. Sci. Adv. 1, e1500222 (2015).

\section{Acknowledgements}

This research was supported by the Basic Science Research Program through the National Research Foundation of Korea (NRF) funded by the Ministry of Education (2019R1I1A1A01061983) and GIST Research Institute (GRI) grant funded by the GIST in 2022. 


\section{Author contributions}

M.K. and Sa.K conceived the idea. M.K performed numerical calculations under the supervision of Sa.K and So.K. All authors discussed the results and contributed to the writing of the manuscript.

\section{Competing interests}

The authors declare no competing interests.

\section{Additional information}

Correspondence and requests for materials should be addressed to S.K. or S.K.

Reprints and permissions information is available at www.nature.com/reprints.

Publisher's note Springer Nature remains neutral with regard to jurisdictional claims in published maps and institutional affiliations.

(c) (i) Open Access This article is licensed under a Creative Commons Attribution 4.0 International License, which permits use, sharing, adaptation, distribution and reproduction in any medium or format, as long as you give appropriate credit to the original author(s) and the source, provide a link to the Creative Commons licence, and indicate if changes were made. The images or other third party material in this article are included in the article's Creative Commons licence, unless indicated otherwise in a credit line to the material. If material is not included in the article's Creative Commons licence and your intended use is not permitted by statutory regulation or exceeds the permitted use, you will need to obtain permission directly from the copyright holder. To view a copy of this licence, visit http://creativecommons.org/licenses/by/4.0/.

(c) The Author(s) 2022 